\title{
On Application of Virtual Reality Technology in Mechanical Course Teaching
}

\author{
Junjian Dang \\ Yantai Nanshan University, Yantai, Shandong, 265713, China
}

Key words: Virtual reality technology, Mechanical course teaching, Specific application

\begin{abstract}
Mechanical course contains a complex internal structure. If daily teaching adopts traditional process, it is difficult to stimulate the interest of students and students may feel dull. At the same time, the mechanical device has a complex and compact body structure, and it is difficult to grasp the fundamental mechanisms. If adopting new kind of virtual reality technology, normal teaching of mechanical course will have aging and realistic features, but also reduce the overall cost of teaching. Virtual reality technology has a database, processing image and graphics in order to depict course scenes in the simulation state. For this, this paper analyze the virtual reality technology mechanical course can select and explore its real application in usual teaching.
\end{abstract}

\section{Introduction}

Mechanical major is concerned with much connotation, and wide scope of knowledge. In traditional steps, such course teaching includes explanation and objects presentation at various stages, relying on multimedia and flipchart for present intrinsic mechanical architecture. Such major is monotonous and abstract, and effectiveness cannot be obtained simply by explanation and passive acceptance. Virtual reality is rooted in computer processing, for the creation of the realistic atmosphere in the simulation state. By means of the perceptible equipment, build the reality. Virtual reality integrates multi-senses, breaking the space barrier in the existing environment. Its application may break through dull atmosphere during conventional teaching, change teaching steps and make students easier to understand, to enable students to understand the unique interest of the mechanical course.

\section{New Virtual Reality Technology}

\section{Analysis on technology connotation}

Virtual reality technology can be used for the creation of virtual environment, and the simulation system works by means of a microcomputer. To create simulated atmosphere and experience this atmosphere, virtual reality integrates interactive information under the condition of multiple sources. Select the real scene within the dynamic framework, and follow this mechanism to create simulation system. Adoption of virtual technology allows users to understand the real atmosphere and scene. With the progress of technology, various refining virtual reality technologies tend to become mature. From a fundamental point of view, the virtual reality sets human-computer interaction, widely used for a variety of existing fields. Interactive and immersive features and endless association are blended into virtual reality ${ }^{[1]}$. The system supports interaction, and presents the value of the subject in the authentic atmosphere.

In the field of technology, virtual reality is increasingly exposed to public attention, which accelerates its original advance rate. Virtual reality relies on the convergence of the microcomputer interface, integrates auditory, visual and tactile senses, and within each period dynamically regulates 
users' behavior. Exposure to the atmosphere can help students to understand the true feelings. The virtual environment and the corresponding body can exchange information and deliver more information in multi-dimension. Get objective information, collect and integrate the information in quantitative and qualitative form, and obtain a deeper understanding on the objective representation, which deepens the original cognition and conception.

\section{New advantages of technology}

New virtual reality technology can be used to build up curriculum system, allowing students to immerse themselves in the created atmosphere and imitating the reality. Teaching technology necessary for virtual reality excludes the preferential dependent trend, breaks the conventional curricular explanation, expands the original two-dimension class, and adds three-dimension new atmosphere. After such modifications, interactive learning replaces original rigid and passive perception, to help students realize the deep teaching connotation, and to the fullest extent, mobilize the potential of cognitive autonomy. At the same time, virtual reality helps nurture higher-level hands-on skills, and adds innovative content. Selected curriculum connotation adds authenticity, flexibility and animation. In detail, virtual reality highlights the following advantages.

First, add new teaching connotation. Mechanical subjects show the strong comprehensive attribute, integrating into usual testing, manufacturing and electrical technology and hydraulic technology, etc. With monotonous explanation, deep connotation cannot be understood, which is urgent to be improved. With virtual means, wholly observe audio images and equipment in the combined form. Virtual reality assists teachers and students to look through the given model, and analyze the normal operation mechanism of the equipment. Experimental teaching draws the following conclusions: curricular means virtual reality screens create more realistic novel atmosphere, to enhance the effectiveness of teaching acquisition ${ }^{[2]}$.

Second, simulate practice processes. For machinery equipment, various machinery manufacturing, and application of sensors, these processes are equipped with high standard curricular practices. In case of the lack of practice, the necessary level of understanding will be reduced and it is not easy to resolve real doubts. Virtual teaching builds a more complete new system. Virtual environment can be used for daily operations, deepening of the curricular practice. At the same time, virtual technology saves more cost, obtains excellent effectiveness and gets rid of various types of operations with dangers.

Third, initiate strong interest of cognition. Mechanical course adopts supporting perceptual system, and has a set of computer control devices. Adopt and retrieve the perceptual system, and integrate supporting display device, driving control device, and voice system. With the help of regulation in comprehensive process, create the virtuality. In a virtual environment, students can personally control selected mechanical equipment, dismantle and try to provide maintenance. Follow-up maintenance becomes easier because immersion in a constructed realistic environment inspires their enthusiasm.

Fourth, virtual teaching corresponds with remote teaching at present. Mechanical course offers remote teaching by means of laboratories within the network framework, and remote teaching has significant virtual property. As open teaching, virtual reality extends the narrow space, and adds human-machine advantage in the complementary state. This modification gets rid of time constraint in long-term state and breaks through the region barrier. The course effectiveness obtained in this way often surpasses the teaching in authentic atmosphere. Students quickly master fundamental experiment mechanism, which is in line with the pre-set research objectives.

\section{Existing Problems in Mechanical Course}

Colleges and universities train talents. Mechanical course is equipped with intrinsic platform. To train higher-level practical talents, the only way is to rely on the new system. Gradually, teaching is improved, and old common types of methods are changed. From the current situation, higher education enters into a new stage today. Mechanical teaching integrates excellent teachers, the environment and equipment, and various colleges and universities focus on such subjects and put 
more energy ${ }^{[3]}$. However, mechanical teaching still has a number of difficulties to be resolved subsequently.

Places teaching can select are still narrow, and sets of devices equipped within the course fail to be timely updated. Limited devices are placed into the site, but students are difficult to clearly observe. Purchase of devices costs much, and there are many types of devices. With science and technology increasingly updating, selected devices are not able to keep up with this trend. Meanwhile, for the workshop teaching, there are still many potential dangers. In case of manipulation mistakes, then sudden failure and even danger are likely to happen.

Subjects of mechanical engineering are related to the larger existing range, and contain much content. In the normal state, through showing physical objects and adopting multimedia to display, students can clearly observe the framework of all kinds of machines. However, many machines have detailed internal components, and only with wall charts and simple analysis, students are not able to deepen understanding. Faced with intense examination pressure, students tend to passively accept, and lose their strong interest in innovation ability, personally handling and practicing they should have. It is urgent to improve inappropriate curricular explanation, and add new contents.

\section{Design New Curriculum System}

\section{Analyze actual needs}

Daily explanation in mechanical course is to analyze the fundamental equipment framework and structure and others, master normal operation mechanism of various components on this basis, learn about unexpected common faults, and select the most appropriate troubleshooting methods. Conventional teaching follows the boring process, and analyzes abstract mechanical content. Students lose their strong interest, and the fundamental motivation cannot be enhanced. By means of curricular explanation, students do not grasp everything provided in the outline. For daily faults, traditional teaching just expresses assumed faults. In contrast, virtual reality relies on the advanced supporting means, simulate and face directly certain component fault ${ }^{[4]}$. Thus, virtual reality is easier to deepen the original perception impression.

\section{Brief system structure}

The new system with virtual reality technology shows interactive and three-dimension properties, in line with the mechanical course. From the perspective of interaction, daily training in the virtual form can adopt immersion method, enhancement method and desktop type, and common distribution method. After measuring the overall cost, curriculum system is set as desktop type. This is because the desktop type adds practical and simple good performance, while reducing the consoles. To design such platform, CAD is equipped with information base in the simulation form, and modeling uses new software. Developed database selects certain virtual environment, and develops simulation training module. Training platform has convenient way to be selected for subsequent operation, to enhance the common features the system displays. Platform offers various training courses able to be flexibly deployed, analyze and summarize all points within the existing outline.

Virtual reality still follows the original outline system, and combines with the true teaching status, properly plans various subjects the exercise presets and highlights the difficulty. For supporting hardware within interaction pathways, human-machine interaction can select the trackball, the handle and the mouse. Console is equipped with the interface input necessary for communication, easy to expand original virtual performance of the system. Microcomputer simulates large-scale and three-dimension complex scenes, and virtual training has better reliability and stability.

\section{Various performance of virtual reality}

First, the virtual classroom shows fundamental mechanical working mechanism. Through switching the viewing angle of each component, students can in detail observe internal chassis, power supply means and hydraulic devices of devices. Chassis system can be subdivided into driving manipulation, 
transmission and steering, and braking components, etc. subdivision and perspective are integrated into one, closely connecting inner complete components and designated control action. This can highlight the visual impression of internal components. Add system roaming, and by means of input control, view the internal form of certain device. For example, for excavators, the system roaming can change the selected path. Observe transmission system, steering and braking devices, and temporarily hide the rest of components. The camera guides the viewing angle at each time point, and repeatedly views internal connection form.

Second, simulate faults, and add follow-up maintenance training. Analyze the typical database faults, and simulate a variety of operations with deviations. System integrates the graphics and acoustic images, and selects the instrumentation necessary for operation. Faced with all kinds of internal and external faults, it can screen the best tools for maintenance. Training process is set to be repetitive, and students are easily familiar with handling steps within each stage.

Third, for dismantling components and installing machine parts, students should be familiar with preset necessary orders. Clarify removal procedure and follow-up installation order and master a number of emphases. Repeated training promotes the real ability of maintenance and operation. In the network state, virtual laboratory has a number of receiving devices, and corresponding output devices, to expand students' horizons and stimulate their potential enthusiasm. Through cultivating a good atmosphere of innovation, students will consciously locate certain problem and then select the appropriate path for analysis, and establish their confidence in innovation.

\section{Specific Application in Teaching}

Simulation assembly gets rid of the common observation program. Preset software the simulation can select, and properly assemble existing system components. Three-dimension modeling can select UG and PRO software, to create model that can be discriminated. Software presets specific assembly sequences, assembly and then detection again. This program simulation follows is for presentation process, in which there is no need of intervention. At the same time, simulation excludes the common variety of limitations and reduces redundant information transmission, which also gets rid of the designated limitations. Three-dimension assembly obtains the prototype able to be observed, and virtual assembly gets assembly process for verification, and sets assembly path to be followed ${ }^{[5]}$.

Evaluation on pre-set innovation program adopts the fundamental design mechanism. Complete the initial selection and the subsequent design. Traditional mechanical design is equipped with a prototype model, and optimized simulation excludes those tedious and redundant steps. For designed assembly layout, the virtual reality properly assesses the movement space and gets rid of the original limitations. Virtual environment is built based on virtual reality assembly technology, and design products follow the same process. Select implementing agencies, make modeling and set simulation analysis in the real state. This integrates the guidance from teachers and reception of students. Digital model follows motion simulation, and then revises the existing analog programs after tested.

Mechanical course focuses on analysis of existing designed products, and pays attention to analysis on design. However, limited by the place and other various factors, conventional techniques cannot clearly present the new machine framework, operating characteristics and so on. Interaction technology of virtual reality has rendering feature, able to change the perspective to facilitate the observation. Teachers and students are immersed and deeply understand the operation process and problems and then carry on innovation and improvement. Close observation can help rationalize innovative ideas the outline, and replace the original plane framework with more vivid three-dimension one.

\section{Conclusions}

From the present situation, it can be seen that daily teaching of mechanical course increasingly accepts new virtual reality technology, and pays attention to nurturing students' interest. Virtual reality has the optimal interaction attribute, able to be used in military training, business and aviation. 
New technology eliminates the shortcomings of traditional classroom, more appropriate and intuitive. Existing practice shows that virtual reality can coordinate all kinds of perception, and the virtual environment built up facilitates the practice. Virtual teaching adds more fun and intuition in teaching process, and harvests expected teaching effectiveness.

\section{References}

[1] Wu Xiangen. On Application of Virtual Reality Technology in "Modern Educational Technology" Course. China Educational Technology, 2011 (03): 96-100.

[2] Zhou Jianzhao, Wang Bin, Hu Chenghe, etc. Applications of Virtual Reality Technology in Mechanical Course Teaching. Mechanical Management and Development, 2011 (03): 177-178 + 183.

[3] Chen Min, Wang Guoyong, Wu Shengnan. Application of Virtual Reality Technology in Mechanical Innovative Experiment Teaching. China Educational Technology Equipment, 2011 (21): 114-116.

[4] Di Kui, Yu Tianbiao, Chen Peiyuan, etc. Application of Virtual Reality Technology in Mechanical Engineering Experiment Teaching. Experimental Technology and Management, 2014 (10): 10-12.

[5] Deng Weimin Application of Virtual Reality Technology in Information Technology Course Teaching. Journal of Sanmenxia Vocational and Technical College, 2015 (03): 59-62. 